\title{
Quantum entanglement of bound particles under free center of mass dispersion
}

\author{
Fernanda Raquel Pinheiro ${ }^{1,2,3 *}$ and A. F. R. de Toledo Piza ${ }^{1}$ \\ ${ }^{1}$ Instituto de Física, Universidade de São Paulo, São Paulo, Brazil \\ ${ }^{2}$ Department of Physics, Stockholm University, 10691 Stockholm, Sweden \\ ${ }^{3}$ NORDITA, 106 91, Stockholm, Sweden
}

\begin{abstract}
On the basis of the full analytical solution of the overall unitary dynamics, the time evolution of entanglement is studied in a simple bipartite model system evolving unitarily from a pure initial state. The system consists of two particles in one spacial dimension bound by harmonic forces and having its free center of mass initially localized in space in a minimum uncertainty wave packet. The existence of such initial states in which the bound particles are not entangled is pointed out. The entanglement of the two particles is shown to be independent of the wavepacket mean momentum, and to increase monotonically in a time scale distinct from that of the spreading of the center of mass wavepacket.
\end{abstract}

\section{Introduction}

Since the early development of Quantum Mechanics, a number of questions have been raised concerning its interpretation and status facing the physical description of reality. 'Entanglement' is one such question, pointed out and developed by Schrödinger [1] in the wake of the paper by Einstein, Podolski and Rosen on the completeness of Quantum Mechanics [2]. The situation considered by Schrödinger in that context hinges on the description of the quantum mechanical state of each one of two separate parts of a composite system, after they have interacted for some time. He observes that even if, prior to the interaction, the state of each of the constituent parts is described by its own quantum state vector (or, in the words of Schrödinger, by its psi-function), in which case the quantum state of the composite system is described by their product, this is in general no longer possible after interaction has run its course, the constituent parts having become 'entangled' by the intervening interaction process. This entanglement is thus associated to the non factorizability of the quantum state of the composite system after the interaction, a trait of quantum mechanics that "enforces its entire departure from classical lines of thought" [1].

After the breakthrough of Bell [3], which opened the way to experimental probing [4] into quantum peculiarities relating to entanglement [2, 5], this concept has become a subject of intense research [6], particularly in connection with the fact that it provides crucial resources for the new research domains of quantum information and quantum

${ }^{*}$ Electronic address: fep@fysik.su.se 
computation [7, 8]. Prominent issues in this context have been, on the one side, developing the characterization and quantification of entanglement, especially as applicable to quantum states which are themselves not represented in terms of a state vector, or a psi-function [6, 9]; and, on the other side, understanding the dynamics of entanglement specially in realistically open systems, i.e. systems undergoing interactions with a complex 'environment' 9. Here one aims particularly at gaining control over the degrading effects of external interactions on the valuable entanglement properties residing in the system of interest. These effects are generally referred to as 'decoherence' processes, and have been given also a positive role in connection with accounting for the practical elusiveness of in principle allowed quantum superpositions of macroscopically distinguishable states [10, 11, i.e., in connection with the 'construction of a classical looking world' on the basis of quantum mechanics.

More recently, investigations of the effect of decoherence processes on the time evolution of entanglement present in a quantum system led to the finding of the rather surprising effect which became known as ESD, "entanglement sudden death" (or, more euphemistically, "early stage disentanglement") [12, which consists in the complete demise, within a finite time, of the entanglement initially present in the system of interest. This effect has in fact been observed in terms of a phenomenological effective, non unitary quantum dynamics which was simulated with photon polarizations in the role of two level systems [13. Subsequently ESD has been also found to be present in extremely simple closed (tripartite) systems evolving unitarily (in fact, multiperiodically) from initially entangled psi-functions [14], showing that environment induced decoherence is not a requisite for this particular phenomenon in the dynamics of entanglement. Furthermore, the fact that one of the subsystems involved in the ESD process does not interact with the remaining parts relativises the role of dynamical correlation processes in the time evolution of entanglement.

This work deals with the dynamics of entanglement in the even simpler context of a bipartite model system which is completely soluble in analytical terms, using tools available in any introductory course on quantum mechanics. The system consists of two particles in one spacial dimension bound by harmonic forces and having its free center of mass initially localized in space in a wave packet of minimum uncertainty. By using center of mass and relative motion dynamical variables, the complete unitary quantum dynamical evolution of this state can be easily written down explicitly. Assuming that the two particles are in a stationary state (e.g., the ground state, for simplicity) of the relative motion, the non stationary character of the initial state reduces therefore just to the dispersive time evolution of the center of mass wavepacket. While the center of mass and relative 'subsystems' are initially not entangled, having their own psi-functions, and remain so at all subsequent times, the same is in general not true when one considers each of the two individual particles as subsystems. It turns out, in fact, that the two particles are in general entangled, and that their entanglement is time dependent as a result of the dispersive time evolution of the center of mass wavepacket. While the presence of entanglement is of no surprise, in view of the fact that the two particles are strongly correlated dynamically by being in a bound state of the harmonic interparticle force, one finds nevertheless that the initial center of mass wavepacket can be prepared so that the two particles are actually not entangled initially. The dispersive time evolution of the center of mass wavepacket is found to induce a monotonic increase in time of the entanglement of the two particles, albeit in a time scale distinct from that associated with the dispersive spreading of the center of mass wavepacket. These facts exemplify in very 
simple terms the surprises and subtleties one may find in the relation of entanglement of subsystems and its dynamics to the unitary dynamical evolution of the system as a whole.

The paper is organized as follows. In section 2 the complete characterization of the model system is given and its dynamics is fully worked out analytically, both in terms of center of mass and relative coordinates and in terms of particle coordinates. The presence of entanglement in the latter description is also discussed. Section 3 constitutes the main body of the work. It is divided in three sub-sections, dealing respectively with the description of the quantum state of entangled subsystems, with some pertinent quantitative measures of entanglement and with a rather detailed discussion of the dynamics of entanglement in the model system. Section 4 contains some concluding remarks. The explicitation of a more technical point relating to the adopted measure of entanglement is given in an Appendix, for completeness.

\section{Alternate descriptions of a flying two-body bound state}

We consider a system consisting of two (spinless) particles in just one dimension, which interact through a translationally invariant attractive potential. If the masses and positions of the particles are $\left\{m_{1}, x_{1}\right\}$ and $\left\{m_{2}, x_{2}\right\}$ respectively and $V\left(x_{2}-x_{1}\right)$ is the potential describing the interaction of the two particles, the dynamics of the system is described by the Hamiltonian

$$
H=\frac{p_{1}^{2}}{2 m_{1}}+\frac{p_{2}^{2}}{2 m_{2}}+V\left(x_{2}-x_{1}\right)
$$

where $p_{1}$ and $p_{2}$ are the momenta canonically conjugated to $x_{1}$ and $x_{2}$. Note that the interaction potential $V$ correlates the dynamics of the two particles. A common simplifying practice in this context is to deal with this correlated two-body problem in terms of an alternate set of dynamical variables describing the center of mass and relative motions rather than in terms of the particle dynamical variables themselves. The reason for this is that in terms of the new variables one has only to deal with two independent, albeit effective, one-body problems. In fact, the appropriate transformations to center of mass variables $X, P$ and relative variables $x, p$ are

$$
R=\frac{m_{1} x_{1}+m_{2} x_{2}}{m_{1}+m_{2}}, \quad P=p_{1}+p_{2} ; \quad r=x_{2}-x_{1}, \quad p=\frac{m_{1} p_{2}-m_{2} p_{1}}{m_{1}+m_{2}},
$$

in terms of which the the Hamiltonian of the two-body problem appears in the guise

$$
H=\frac{P^{2}}{2 M}+\left(\frac{p^{2}}{2 \mu}+V(x)\right) \equiv H_{\mathrm{CM}}+H_{\mathrm{rel}} .
$$

Here the new mass parameters are, respectively, the total mass $M$ and the reduced mass $\mu$ of the particles, which in terms of the particle masses can be expressed as

$$
M=m_{1}+m_{2}, \quad \mu=\frac{m_{1} m_{2}}{m_{1}+m_{2}} .
$$

This reduction procedure can be used both in classical and in quantum contexts. In the former case it involves a canonical transformation, while the latter it involves a unitary transformation of the basic canonical variables. Even though this is in fact rather trivially evident, it is worth noting explicitly that in both cases the system maintains a composite character, but which in the latter rendering reduces to two non-interacting parts. 
We now recall the simple quantum mechanical treatment of the system, taking as a specific instance of the two-body interaction potential a simple harmonic potential $V(x)=$ $\mu \omega^{2} x^{2} / 2$. The stationary solutions of the Schrödinger equation for the composite system are well known and simple to obtain. They consist in the product of a center of mass momentum eigenfunction with a stationary state of the harmonic oscillator equation which governs the dynamics of the relative motion. Taking the relevant state to be the oscillator ground state one has

$$
\Psi(R, r)=e^{i K R} \frac{1}{\pi^{\frac{1}{4}} \sqrt{b}} e^{-\frac{1}{2}\left(\frac{r}{b}\right)^{2}}, \quad b=\sqrt{\frac{\hbar}{\mu \omega}} .
$$

Note that one faces here the well known normalization problem in the case of the continuous spectrum of the center of mass Hamiltonian. We will not have to deal explicitly with this, however, since we consider a (non-stationary) normalized gaussian wavepacket of width $B$ and mean momentum $\hbar K$ as the wavefunction describing the initial state of the center of mass part. The relative part remains in its chosen ground stationary state, so that our normalized non-stationary initial state can be written as

$$
\Psi(R, r)=\frac{1}{\pi^{\frac{1}{4}} \sqrt{B}} e^{-\frac{R^{2}}{2 B^{2}}} e^{i K \cdot R} \frac{1}{\pi^{\frac{1}{4}} \sqrt{b}} e^{-\frac{1}{2}\left(\frac{r}{b}\right)^{2}} .
$$

In order to obtain the time evolution of such a globally non-stationary state it is most convenient to have it rewritten in the momentum representation by means of a double Fourier transform

$$
\Psi(\kappa, \xi)=\int e^{-i \kappa R} e^{-i \xi r} \Psi(R, r) d R d r=4 \sqrt{\frac{B b}{\pi}} e^{-2 B^{2}(\kappa-K)^{2}} e^{-2 b^{2} \xi^{2}} .
$$

Since the relative state is an oscillator eigenstate, the Fourier transform involving the relative coordinate is strictly not necessary for this purpose, but it will be convenient further on for reverting the description to the original particle variables, instead of the center of mass and relative variables.

Time evolution can now be implemented by just bringing in the appropriate timedependent phase factors associated with the different center of mass momentum eigenfunctions as well as with the relative oscillator eigenfunction. This leads to the well known translation and spreading of the center of mass wave packet, with no other effect than an irrelevant overall time-dependent phase factor originating from the internal energy eigenstate. More formally, the time evolution is represented by the action of the evolution operator

$$
U(t, 0)=e^{-\frac{i}{\hbar} \frac{P^{2} t}{2 M}} \otimes e^{-\frac{i}{\hbar} H_{\mathrm{rel}} t},
$$

which reduces to numerical phase factors when acting on the momentum space representation. Thus, after elapsed time $t$, the momentum space wave-function (2.3) becomes

$$
\Psi(\kappa, \xi, t)=4 \sqrt{\frac{B b}{\pi}} e^{-\frac{i \hbar \kappa^{2} t}{2 M}} e^{-2 B^{2}(\kappa-K)^{2}} e^{-i \frac{\omega}{2} t} e^{-2 b^{2} \xi^{2}} .
$$

The simplicity of this result is basically due to the independence of the center of mass and relative dynamics. In particular, the stationary character of the relative motion state implies that its contribution to the joint time-evolution reduces to an overall time dependent phase factor. The time evolution of the center of mass wave packet is 
itself more involved, as different momentum components acquire different time-dependent phases which govern its dispersive displacement. The center of mass and relative motion parts of the composite system have however their independence fully uncompromised by these time-dependences, as clearly exhibited by the factorization of the overall wavefunction into center of mass and relative motion factors at all times.

Now let us return to our former decomposition of the system, in terms of its two constituent particles. In momentum representation, this can be done on the basis of the solution written in eq. (2.4), through reverting to the dynamical variables relating to the individual particles. The appropriate transformation here is (cf. eqs. (2.1))

$$
\kappa=k_{1}+k_{2}, \quad \xi=\frac{m_{1} k_{2}-m_{2} k_{1}}{M},
$$

where $k_{1}$ and $k_{2}$ are the wave numbers associated with the momentum eigenfunctions of particles 1 and 2 respectively. In terms of these, the time evolved state appears as

$$
\Psi\left(k_{1}, k_{2}, t\right)=4 \sqrt{\frac{B b}{\pi}} e^{\frac{-i \hbar t}{2 M}\left(k_{1}+k_{2}\right)^{2}} e^{-2 B^{2}\left(k_{1}+k_{2}-K\right)^{2}} e^{-i \frac{\omega}{2} t} e^{-\frac{2 b^{2}}{M^{2}}\left(m_{1} k_{2}-m_{2} k_{1}\right)^{2}} .
$$

An important feature of this alternate rendering of the state of the two-particle system is that the dependence on $k_{1}$ and on $k_{2}$ does not factor for all times $t>0$, as can be seen by the occurrence of terms involving the product $k_{1} k_{2}$ in the exponents. Note however that factorization will in fact occur at $t=0$ for a particular choice of width parameter $B$ of the center of mass wavepacket. In fact, when this parameter is tuned to the value satisfying

$$
B^{2} \rightarrow B_{0}^{2}=\frac{m_{1} m_{2}}{M^{2}} b^{2}=\frac{\mu}{M} b^{2}
$$

these non-factoring terms drop out, and one obtains a product wavefunction also in terms of the individual particle variables. This is clearly a result particular to our choice of a real gaussian envelope function for the center of mass wavepacket, combined with the gaussian form of the relative ground state in the harmonic particle-particle interaction potential.

The factorizability of the wavefunction (2.4) at all times $t$ allows for associating the quantum state of the center of mass and relative parts of the system with a respective factor of that wavefunction. Each of these two parts is therefore endowed with its own independent quantum mechanical probability amplitude. Non factorizability when it is expressed in terms of particle variables as in eq. (2.5), on the other hand, implies that the joint probability amplitude includes such correlation involving the two particles which impedes an independent description of each one of them in terms of probability amplitudes. This is just what has been called by Schrödinger (in 1935, [1]) the quantum 'entanglement' of the two particles.

Our findings so far indicate moreover that a definite distinction should be made between entanglement and correlations between parts of the composite system. In fact, in the state described by eqs. (2.4) and (2.5), the two particles are, at all times, and in particular at $t=0$, strongly correlated by finding themselves bound in the stationary ground state of relative motion. The initial state factorization in terms of particle variables for $B=B_{0}$ provides thus for a counter example disallowing direct association of such correlation with entanglement. The factorized amplitudes of the wavefunction cast in terms of the particle variables, eq. (2.5), in a generic factorized instance $B^{2}=\mu b^{2} / M$ at $t=0$, appear as

$$
\psi_{1}\left(k_{1}\right)=N_{1} e^{-2 b^{2} \frac{\mu}{M}\left(1+\frac{m_{2}}{m_{1}}\right) k_{1}^{2}+4 b^{2} \frac{\mu}{M} k_{1} K} \quad \text { and } \quad \psi_{2}\left(k_{2}\right)=N_{2} e^{-2 b^{2} \frac{\mu}{M}\left(1+\frac{m_{1}}{m_{2}}\right) k_{2}^{2}+4 b^{2} \frac{\mu}{M} k_{2} K},
$$


$N_{1}$ and $N_{2}$ being the appropriate normalization constants. It is apparent in these expressions that, while the quantum kinematical property of amplitude factorization ensures lack of entanglement, each one of the two factor amplitudes involve parameters referring to the two particles and bearing on their dynamical correlation.

\section{Quantum states of entangled particles}

According to the rules of quantum mechanics, knowledge of the joint wavefunction (2.5) provides us with the most complete information one can obtain about the system itself. It must then, in particular, also provide us with the most complete information one can obtain about each of its constituent particles, considered separately. The formal tool usually employed to extract these more restricted pieces of information from the amplitude (2.5) describing the quantum state of the two-particle system involves first setting up the so called density matrix, defined as

$$
\rho\left(k_{1}, k_{2} ; k_{1}^{\prime}, k_{2}^{\prime} ; t\right) \equiv \Psi\left(k_{1}, k_{2}, t\right) \Psi^{*}\left(k_{1}^{\prime}, k_{2}^{\prime}, t\right) .
$$

The 'diagonal' part of this, i.e., the part with $k_{1}=k_{1}^{\prime}$ and $k_{2}=k_{2}^{\prime}$, corresponds just to the joint probability distribution (in momentum space) of the two particle system, at time $t$. Moreover, from the normalization of the wavefunction $\Psi\left(k_{1}, k_{2}, t\right)$ it follows that

$$
\int d k_{1}^{\prime \prime} \int d k_{2}^{\prime \prime} \rho\left(k_{1}, k_{2} ; k_{1}^{\prime \prime}, k_{2}^{\prime \prime} ; t\right) \rho\left(k_{1}^{\prime \prime}, k_{2}^{\prime \prime} ; k_{1}^{\prime}, k_{2}^{\prime} ; t\right)=\rho\left(k_{1}, k_{2} ; k_{1}^{\prime}, k_{2}^{\prime} ; t\right) .
$$

The double integration works here just as a matrix multiplication, so that this relation in fact shows that, considered as representing an operator, the density matrix (3.7) associated with the normalized wavefunction (2.5) is hermitian and idempotent, and therefore a projection operator.

Now the mean value of any observable $\mathcal{O}^{(1)}$ pertaining to the first particle can be obtained in terms of the density matrix as

$$
\begin{aligned}
\left\langle\mathcal{O}^{(1)}\right\rangle_{t} & =\int d k_{1} \int d k_{1}^{\prime} \mathcal{O}_{k_{1}^{\prime} k_{1}}^{(1)} \int d k_{2} \rho\left(k_{1}, k_{2} ; k_{1}^{\prime}, k_{2} ; t\right) \equiv \\
& \equiv \int d k_{1} \int d k_{1}^{\prime} \mathcal{O}_{k_{1}^{\prime} k_{1}}^{(1)} \bar{\rho}^{(1)}\left(k_{1}, k_{1}^{\prime} ; t\right)
\end{aligned}
$$

where $\mathcal{O}_{k_{1}^{\prime} k_{1}}^{(1)}$ is the momentum representation of the observable under consideration, $\mathcal{O}^{(1)}$. Since this works for any observable pertaining to particle 1, it follows that the object

$$
\bar{\rho}^{(1)}\left(k_{1}, k_{1}^{\prime} ; t\right) \equiv \int d k_{2} \rho\left(k_{1}, k_{2} ; k_{1}^{\prime}, k_{2} ; t\right),
$$

known as the reduced density matrix for particle 1 contains all information concerning this particle, when considered independently of the rest of the system. The operation leading to the reduced density matrix is usually referred to as taking a partial trace of the density matrix $\rho\left(k_{1}, k_{2} ; k_{1}^{\prime}, k_{2}^{\prime} ; t\right)$ over particle 2 , and amounts to an all inclusive acceptance of whatever properties it would have correlated to the particle under scrutiny. A similar argument allows one to collect all information concerning particle 2 which is similarly inclusive with respect to properties of particle 1 , by taking a partial trace of $\rho\left(k_{1}, k_{2} ; k_{1}^{\prime}, k_{2}^{\prime} ; t\right)$ over this particle, i.e. 


$$
\bar{\rho}^{(2)}\left(k_{2}, k_{2}^{\prime} ; t\right) \equiv \int d k_{1} \rho\left(k_{1}, k_{2} ; k_{1}, k_{2}^{\prime} ; t\right)
$$

Note that, again in view of the normalization of the wavefunction (2.5), one has

$$
\int d k_{1} \bar{\rho}^{(1)}\left(k_{1}, k_{1} ; t\right)=\int d k_{2} \bar{\rho}^{(2)}\left(k_{2}, k_{2} ; t\right)=1 .
$$

An application of these ideas to the specific case of the two-particle system as considered here goes as follows. First, the density matrix reads explicitly (cf. eqs. (2.5) and (3.7))

$$
\begin{aligned}
\rho\left(k_{1}, k_{1}^{\prime}, k_{2}, k_{2}^{\prime}, t\right)= & \frac{16 B b}{\pi} e^{\left.\frac{-i \hbar t}{2 M}\left(\left(k_{1}+k_{2}\right)^{2}-\left(k_{1}^{\prime}+k_{2}^{\prime}\right)^{2}\right)\right)} e^{-2 B^{2}\left(\left(k_{1}+k_{2}-K\right)^{2}+\left(k_{1}^{\prime}+k_{2}^{\prime}-K\right)^{2}\right)} \times \\
& \times e^{-\frac{2 b^{2}}{M^{2}}\left(\left(m_{1} k_{2}-m_{2} k_{1}\right)^{2}+\left(m_{1} k_{2}^{\prime}-m_{2} k_{1}^{\prime}\right)^{2}\right)}
\end{aligned}
$$

Next, the reduced density matrix for particle 1 can be obtained by taking a partial trace of this over particle 2, which amounts here to setting $k_{2}=k_{2}^{\prime}$ and performing a gaussian integration over the momentum variable $k_{2}$. This gives the somewhat lengthy result

$$
\begin{aligned}
& \rho^{(1)}\left(k_{1}, k_{1}^{\prime} ; t\right)= \\
& \quad=\frac{8 B b M e^{-\frac{4 K^{2} B^{2} b^{2} m_{1}^{2}}{M^{2} B^{2}+m_{1}^{2} b^{2}}}}{\pi \sqrt{M^{2} B^{2}+m_{1}^{2} b^{2}}} e^{-\frac{\hbar^{2} t^{2}}{16\left(M^{2} B^{2}+m_{1}^{2} b^{2}\right)}\left(k_{1}-k_{1}^{\prime}\right)^{2}} e^{4 K B^{2}\left(k_{1}-k_{1}^{\prime}\right)} e^{\frac{\left(\mu b^{2}-M B^{2}\right)^{2}}{M^{2} B^{2}+m_{1}^{2} b^{2}}\left(k_{1}+k_{1}^{\prime}\right)^{2}} \times \\
& \quad \times e^{2 K B^{2} M\left(\frac{\mu b^{2}-M B^{2}}{M^{2} B^{2}+m_{1}^{2} b^{2}}\right)\left(k_{1}+k_{1}^{\prime}\right)} e^{-\frac{2}{M^{2}}\left(M^{2} B^{2}+m_{2}^{2} b^{2}\right)\left(k_{1}^{2}+k_{1}^{\prime 2}\right)} e^{-\frac{i \hbar t}{2}\left(\frac{m_{1} b^{2}}{M^{2} B^{2}+m_{1}^{2} b^{2}}\right)\left(k_{1}^{2}-k_{1}^{\prime 2}\right)}
\end{aligned}
$$

In view of the complete symmetry of the density matrix (3.9) under an interchange of indices $1 \leftrightarrow 2$, the reduced density matrix for particle 2 can be immediately read off from eq. (3.10) simply by replacing all indices 1 by 2 and vice versa.

\subsection{Reduced density factorization and entanglement}

An important feature of the reduced density matrix (3.10) and also of its companion $\rho^{(2)}\left(k_{2}, k_{2}^{\prime} ; t\right)$ is that their dependence on primed and unprimed variables does not factor in general. This is in fact clear in view of the exponentials involving $\left(k_{i} \pm k_{i}^{\prime}\right)^{2}$. Nonfactorizability implies that the reduced densities cannot be obtained from single quantum amplitudes in the same way that the full density matrix (3.7) is obtained from the wavefunction (2.5), and indicates the entanglement of the two particle system in the selected quantum state 1 . The reduced densities are however hermitian objects in the sense that

$$
\rho^{(i) *}\left(k_{i}, k_{i}^{\prime} ; t\right)=\rho^{(i)}\left(k_{i}^{\prime}, k_{i} ; t\right), \quad i=1,2 .
$$

A useful representation of the reduced densities can therefore be obtained in terms of the solution of the eigenvalue problem

\footnotetext{
${ }^{1}$ Recall that factorization may occur in special cases, such as the one pointed out earlier (see eq. (2.6)) in which the wavefunction (2.5) factors, namely $t=0$, and $B \rightarrow B_{0}=b \sqrt{\mu / M}$. In this particular situation it is possible to associate probability amplitudes to each of the two particles, although each of the amplitudes will involve the mass parameters of both particles, in addition to 'shared' parameters such as the oscillator parameter $b$ and the width $B_{0}$ of the center of mass wavepacket.
} 


$$
\int \rho^{(i)}\left(k_{i}, k_{i}^{\prime} ; t\right) \chi_{n}^{(i)}\left(k_{i}^{\prime}, t\right) d k_{i}^{\prime}=\lambda_{n}^{(i)}(t) \chi_{n}^{(i)}\left(k_{i}, t\right)
$$

Hermiticity ensures that the eigenvalues are real and the eigenfunctions can be made orthonormal. In addition, from the fact that the mother density (3.7) is a projection operator, one can actually deduce that the eigenvalues are in fact non negative, i.e. $\lambda_{n}^{(i)}(t) \geq 0$. The reduced density $\rho^{(i)}\left(k_{i}, k_{i}^{\prime} ; t\right)$ can therefore be written in the form

$$
\rho^{(i)}\left(k_{i}, k_{i}^{\prime} ; t\right)=\sum_{n} \lambda_{n}^{(i)}(t) \chi_{n}^{(i)}\left(k_{i}, t\right) \chi_{n}^{(i) *}\left(k_{i}^{\prime}, t\right)
$$

which engages all the (orthonormal) eigenfunctions with nonvanishing eigenvalue. Note also that, in view of the normalization property (3.8), one has $\sum_{n} \lambda_{n}^{(i)}(t)=1$.

A rather non trivial outcome of this representation is that the two reduced densities originating from a given mother density matrix of the form (3.7) have the same eigenvalues, their eigenfunctions being related as

$$
\chi_{n}^{(2)}\left(k_{2}, t\right)=N_{n}(t) \int d k_{1} \chi_{n}^{(1) *}\left(k_{1}, t\right) \Psi\left(k_{1}, k_{2}, t\right)
$$

where $N_{n}(t)$ is an appropriate normalization factor. Furthermore, the joint wavefunction itself may be written in terms of the reduced densities eigenfunctions as (see Appendix))

$$
\Psi\left(k_{1}, k_{2}, t\right)=\sum_{n} \sqrt{\lambda_{n}(t)} \chi_{n}^{(1)}\left(k_{1}, t\right) \chi_{n}^{(2)}\left(k_{2}, t\right),
$$

in what is usually referred to as the Schmidt decomposition [8].

Therefore, what one can do in general is to write the reduced densities as linear combinations of orthogonal projection operators with non negative coefficients of unit sum, also known as convex sums of orthogonal projection operators. Note that cases in which dependence on primed and unprimed variables factors in the reduced densities are duly covered as cases in which a single nonvanishing, hence unit valued, eigenvalue occurs in eq. (3.11). This makes the reduced densities themselves projection operators.

Whenever the convex sum (3.11) involves more than a single term the same happens with eq. (3.12), which reveals a connection of non factorization of the dependence on primed and unprimed variables in the reduced densities and the non factorization of the dependence on $k_{1}$ and $k_{2}$ in the wavefunction of the composite system. This is precisely the technical characterization of entanglement given by Schrödinger in his paper of 1935 [1. Thus, entanglement is revealed in this case by the fact that the reduced densities have more than a single nonvanishing eigenvalue (hence needing more than a single eigenfunction for its representation). In view of eq. (3.12) one can see this as scars left from the process of 'orphaning' of quantum amplitude correlations implied by multiple terms in eq. (3.12).

Finally, it is worth stressing that the preceding analysis has been performed on objects ultimately related to the wavefunction of the composite system at a given time $t$. Therefore all the derived quantities are in general time dependent in a way which can be ultimately traced back to the unitary time evolution of the wavefunction of the composite system. In particular, if one devises a quantitative measure of entanglement, one will be able to follow its evolution in time. 


\subsection{A simple quantitative measure of entanglement}

The coefficients in the convex sum over orthogonal projection operators in eq. (3.11), given the condition $\sum_{n} \lambda_{n}(t)=1$ which follows from normalization of the wavefunction of the composite system, can be seen as a probability distribution over the set of projections onto the eigenvectors of the reduced density. Furthermore, as the occurrence of more than a single nonvanishing weight signals entanglement of the two parts of the considered system which are under consideration, one is led to the use of entropy-like functions as they are used in statistical mechanics to provide for quantification of the degree of entanglement. A common choice for that is the so called von Neumann entropy [15, 6], given as

$$
S\left[\rho^{(1)}(t)\right]=-\sum_{n} \lambda_{n} \log \lambda_{n}
$$

This is a function endowed both with additivity and convexity properties [16, but its evaluation is often more costly than alternate functions, such as the so called '2-Rényi' entropy $S_{2 R}$ and the 'linear' entropy $\Delta[\underline{6}$, which are defined respectively as

$$
S_{2 R}\left[\rho^{(1)}(t)\right]=-\log \sum_{n} \lambda_{n}^{2} \quad \text { and } \quad \Delta\left[\rho^{(1)}(t)\right]=\sum_{n} \lambda_{n}\left(1-\lambda_{n}\right)=1-\sum_{n} \lambda_{n}^{2} .
$$

The evaluation of the sum appearing in these objects can be carried out in a simple way in terms the reduced density matrix itself, by noting that

$$
\sum_{n} \lambda_{n}^{2}=\operatorname{Tr}\left[\rho^{(1)}(t)\right]^{2}=\int d k_{1} \int d k_{2} \rho^{(1)}\left(k_{1}, k_{2} ; t\right) \rho^{(1)}\left(k_{2}, k_{1} ; t\right) .
$$

An identical result is obtained if the reduced density $\rho^{(2)}\left(k_{1}, k_{2} ; t\right)$ is used instead of $\rho^{(1)}\left(k_{1}, k_{2} ; t\right)$.

Both of these functions are non-negative and have the desirable convexity properties, although the linear entropy is not additive. This fact is however of little relevance in this context, and since, unlike the values of $S_{2 R}$, the values of $\Delta$ are bounded above by 1 , we will use the linear entropy for quantifying the entanglement of the two particles.

Evaluation of the linear entropy for the reduced density obtained in eq. (3.10) gives

$$
\begin{aligned}
\Delta(t)=1-\operatorname{Tr}\left[\rho^{(1)}(t)\right]^{2} & =1-\frac{1}{\sqrt{\frac{\hbar^{2} t^{2}}{16 M^{2} B^{2} b^{2}}+\frac{\left(M^{2} B^{2}+m_{1}^{2} b^{2}\right)\left(M^{2} B^{2}+m_{2}^{2} b^{2}\right)}{M^{4} B^{2} b^{2}}}} \equiv \\
& \equiv 1-\frac{b}{B} \frac{\tau_{B}}{\tau} \frac{1}{\sqrt{1+\frac{t^{2}}{\tau^{2}}}} .
\end{aligned}
$$

In the last expression, $\tau_{B}=M B^{2} / \hbar$ is the characteristic time associated with the dispersive spreading of the center of mass wavepacket, and $\tau$ has been introduced as the characteristic time associated with the time dependence of the linear entropy. It is given by

$$
\tau \equiv \tau_{B} \sqrt{\left(1+\frac{m_{1}^{2} b^{2}}{M^{2} B^{2}}\right)\left(1+\frac{m_{2}^{2} b^{2}}{M^{2} B^{2}}\right)},
$$

an expression which is symmetric under the interchange of indices $1 \leftrightarrow 2$, consistently with the fact that the same result for the linear entropy is obtained if one uses $\rho^{(2)}\left(k_{2}, k_{2}^{\prime}, t\right)$, instead of $\rho^{(1)}\left(k_{1}, k_{1}^{\prime}, t\right)$, in its evaluation. 


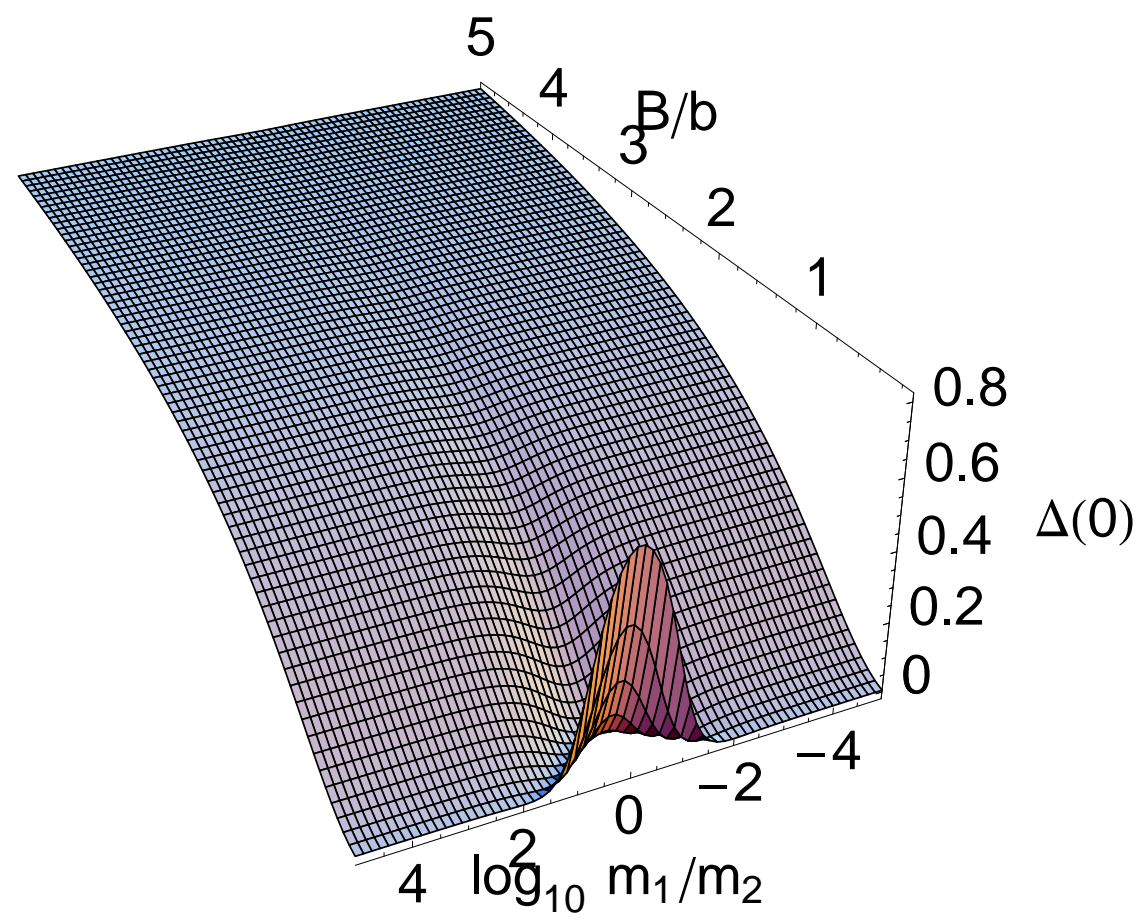

Figure 1: Overall view of the initial linear entropy landscape as a function of $\log _{10} m_{1} / m_{2}$ and $B / b$. The logarithmic scale for the mass ratio emphasizes the symmetry under interchange of $m_{1}$ and $m_{2}$. Most interesting features are seen to develop along the plane $m_{1} / m_{2}=1\left(\log _{10} m_{1} / m_{2}=0\right)$, mainly around $B / b=0.5$.

\subsection{Initial entanglement and its dynamics}

A first, general feature to be noted in connection with the expression obtained for the linear entropy $\Delta(t)$ is that it turns out to be independent of the mean center of mass momentum $K$. This indicates Galilean invariance of the dynamics of entanglement in the present case, and is similar to what happens in connection with the dispersive spreading of wavepackets in position space. Actually this feature can be examined in very simple terms by considering the action of the center of mass momentum translation operator

$$
G(\zeta)=e^{i \zeta X}=e^{i \zeta \frac{m_{1} x_{1}+m_{2} x_{2}}{M}}
$$

on the representation of the wavefunction of the composite system given in eq. (3.12). The fact that the two position operators $x_{1}$ and $x_{2}$ commute allows for the factorization of $G(\zeta)$ as $G_{1}(\zeta) \otimes G_{2}(\zeta)$, so that

$$
G(\zeta) \Psi\left(k_{1}, k_{2}, t\right)=\sum_{n} \sqrt{\lambda_{n}}\left(G_{1}(\zeta) \chi_{n}^{(1)}\left(k_{1}, t\right)\right)\left(G_{2}(\zeta) \chi_{n}^{(2)}\left(k_{2}, t\right)\right) .
$$

The center of mass momentum translation therefore does not affect the amplitudes $\sqrt{\lambda_{n}}$ (also known as the coefficients of the Schmidt decomposition) which govern the entanglement. The eingenvalues of the reduced densities are therefore preserved, and so is, in particular, the value of the linear entropy.

Consider next the value of the reduced entropy for the class of initial states of the form 


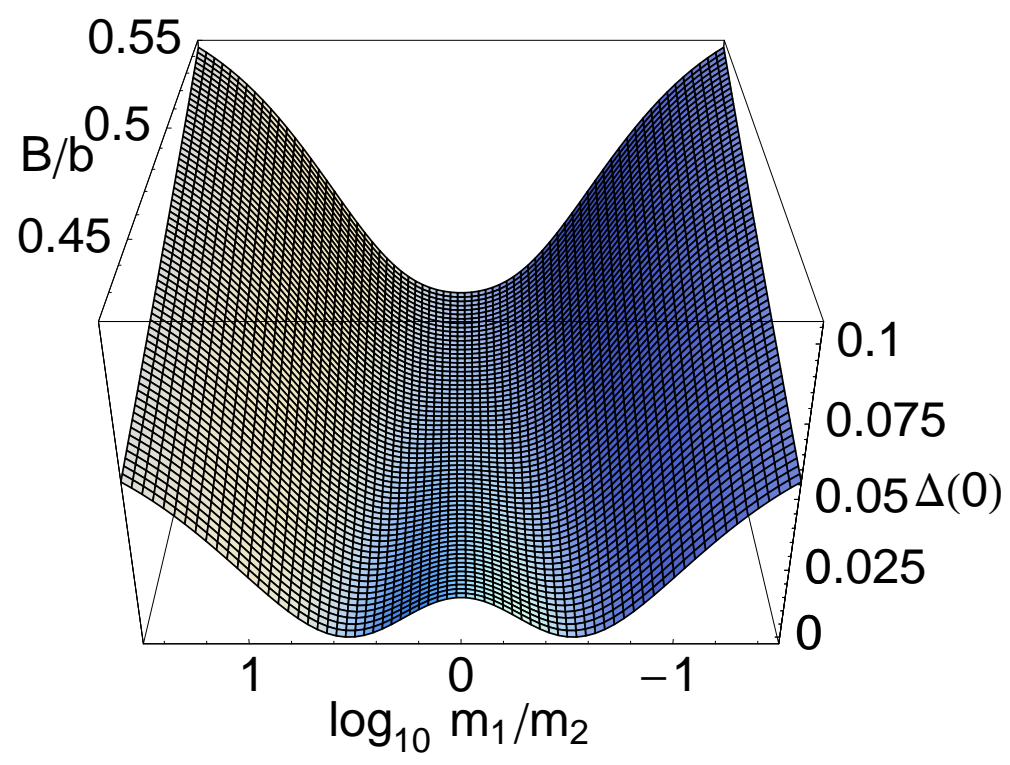

Figure 2: Closer view of the most interesting section of the initial linear entropy landscape as a function of $\log _{10} m_{1} / m_{2}$ and $B / b$. The section shown here contains the point $m_{1} / m_{2}=1$, $B / b=0.5$ where the valley of $\Delta(t=0)$ at $m_{1} / m_{2}=1$ for $B / b>0.5$ branches into two valleys where $\Delta(0)=0$, separated by a ridge still at $m_{1} / m_{2}=1$, for $B / b<0.5$.

(2.3). It is given in terms of the the mass parameters $m_{1}, m_{2}$ and of the gaussian width parameters $b, B$ as

$$
\begin{aligned}
\Delta_{0} \equiv \Delta(t=0)=1-\frac{b}{B} \frac{\tau_{B}}{\tau} & =1-\frac{b}{B}\left[\left(1+\frac{m_{1}^{2} b^{2}}{M^{2} B^{2}}\right)\left(1+\frac{m_{2}^{2} b^{2}}{M^{2} B^{2}}\right)\right]^{-\frac{1}{2}}= \\
& =1-\left[\frac{B^{2}}{b^{2}}\left(1-\frac{b^{2}}{B^{2}} \frac{m_{1} / m_{2}}{\left(1+m_{i} / m_{2}\right)^{2}}\right)^{2}+1\right]^{-\frac{1}{2}}
\end{aligned}
$$

As shown, it can be expressed in terms of just the two dimensionless quantities $B / b$ and $m_{1} / m_{2}$. Their value, supplemented by one additional scale setting quantity, which can be conveniently taken to be the total mass $M$, completely determines particular initial states of the assumed form. Note that the linear entropy is independent of the scale setting, so that its behavior over different domains in the manifold of states of the form (2.3) may be mapped by analyzing $\Delta_{0}$ as a function of $B / b$ and $m_{1} / m_{2}$. An overall view of the behavior of this function can be seen as a surface plot in fig. 1.

We now discuss some features of this initial entanglement landscape which can be scrutinized by analyzing eq. (3.15) in some detail. First of all, one easily finds that, for any given value of the mass ratio $m_{1} / m_{2}$, the relevant extremum of $\Delta_{0}$ as a function of $B / b$ occurs for $B / b \rightarrow \sqrt{m_{1} m_{2}} /\left(1+m_{1} / m_{2}\right)$. Substitution back into the expression for $\Delta_{0}$ shows that one has at these points $\Delta_{0}=0$. We thus re-obtain, in the guise of the vanishing of the initial value of the linear entropy, the parametric conditions for the factorization of the initial wavefunction when cast in terms of particle variables.

All the possible values of $B / b$ associated with this set of unentangled initial states lie in the interval $0 \leq B / b \leq 0.5$, as follows from the range available for $m_{1} / m_{2}$, namely 


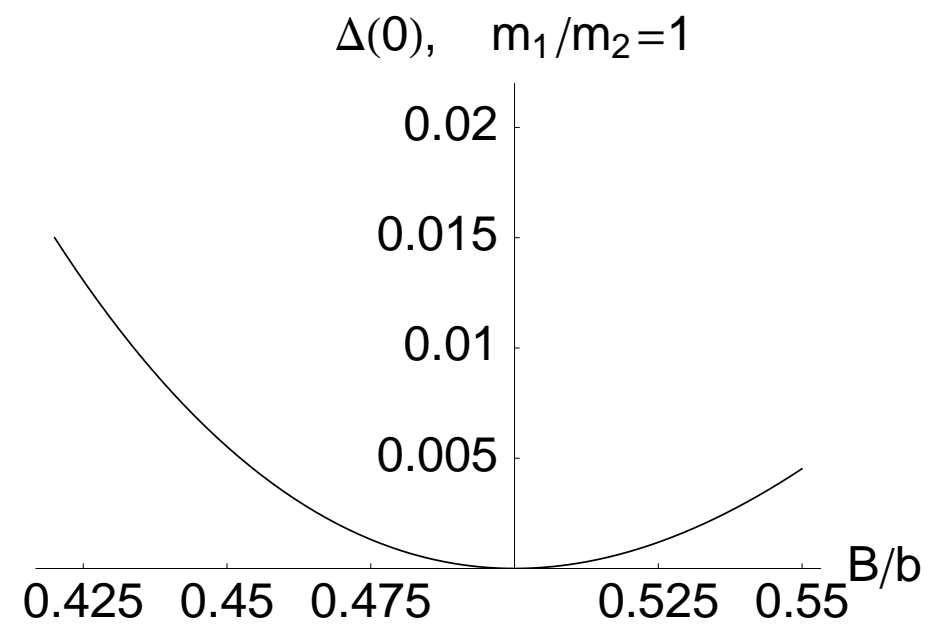

Figure 3: A cut of the surface shown in fig. 22 at the plane $\alpha=m_{1} / m_{2}=1$, showing values of the maxima (for $\beta<0.5$ ) and minima (for $\beta>0.5$ ) of the initial linear entropy. The plotted range of $\beta=B / b$ is the same as in fig. 2 ,

$0 \leq m_{1} / m_{2}<\infty$. They are therefore confined to a front vertical slice of fig. 1, In order to explore the behavior of the initial value of the linear entropy for $B / b>0.5$ one may fix the value of this variable and study the dependence of the initial linear entropy on $m_{1} / m_{2}$. What one finds in this way is that there is an extremum at $m_{1} / m_{2}=1$ for all possible values of $B / b$, in addition to two other extrema with $m_{1} / m_{2}$ values given by

$$
\left(\frac{m_{1}}{m_{2}}\right)_{ \pm}=\frac{b^{2}}{2 B^{2}}\left(1 \pm \sqrt{1-4 B^{2} / b^{2}}\right)-1
$$

which are real, non-negative numbers only for $B / b \leq 0.5$. It turns out that the extremum at $m_{1} / m_{2}=1$ is in fact a (nonvanishing) minimum of the initial linear entropy for $B / b>$ 0.5 , which however becomes a maximum for $B / b<0.5$. In this range, the additional extrema are the minima for which $\Delta(0)=0$, which have already been identified earlier. The fact that there are two such minima is due to the symmetry of the linear entropy under interchange of the masses $m_{1}$ and $m_{2}$. As a check on this, one can easily verify that $\left(m_{1} / m_{2}\right)_{-}\left(m_{1} / m_{2}\right)_{+}=1$ for all values of $B / b$. At $B / b=0.5, \Delta(0)$ has a very flat minimum (a zero of the fourth order) as a function of $m_{1} / m_{2}$ at $m_{1} / m_{2}=1$. These features are displayed in fig. 2, which covers the relevant domain of the initial linear entropy landscape, namely $0.031 \leq m_{1} / m_{2} \leq 31$ and $0.42 \leq B / b \leq 0.55$. A cut of this surface graph, showing the values of $\Delta(0)$ at $m_{1} / m_{2}=1$ as a function of $B / b$ is shown in fig. 3. In this graph the ranges $B / b<0.5$ and $B / b<0.5$ correspond respectively to a ridge and to a valley in the $\Delta(0)$ surface.

The time dependence of the linear entropy measure of particle-particle entanglement consists in a simple, monotonic approach to the upper bound $\Delta=1$ as seen in eq. (3.13). It involves an algebraic expression which is of the same form as that which governs the dispersive spreading of the center of mass wavepacket albeit involving a time scale $\tau \neq \tau_{B}$. Since $\tau_{B}$ is the relevant time scale in the unitary dynamical evolution of the initial state of the composite system, it is both natural and convenient to use this time scale to analyze the dependence of the characteristic time $\tau$ of the dynamics of entanglement on the different initial states of the form (2.3).

The quantity $\tau / \tau_{B}$ given in eq. (3.15) is seen to be closely related to the initial 


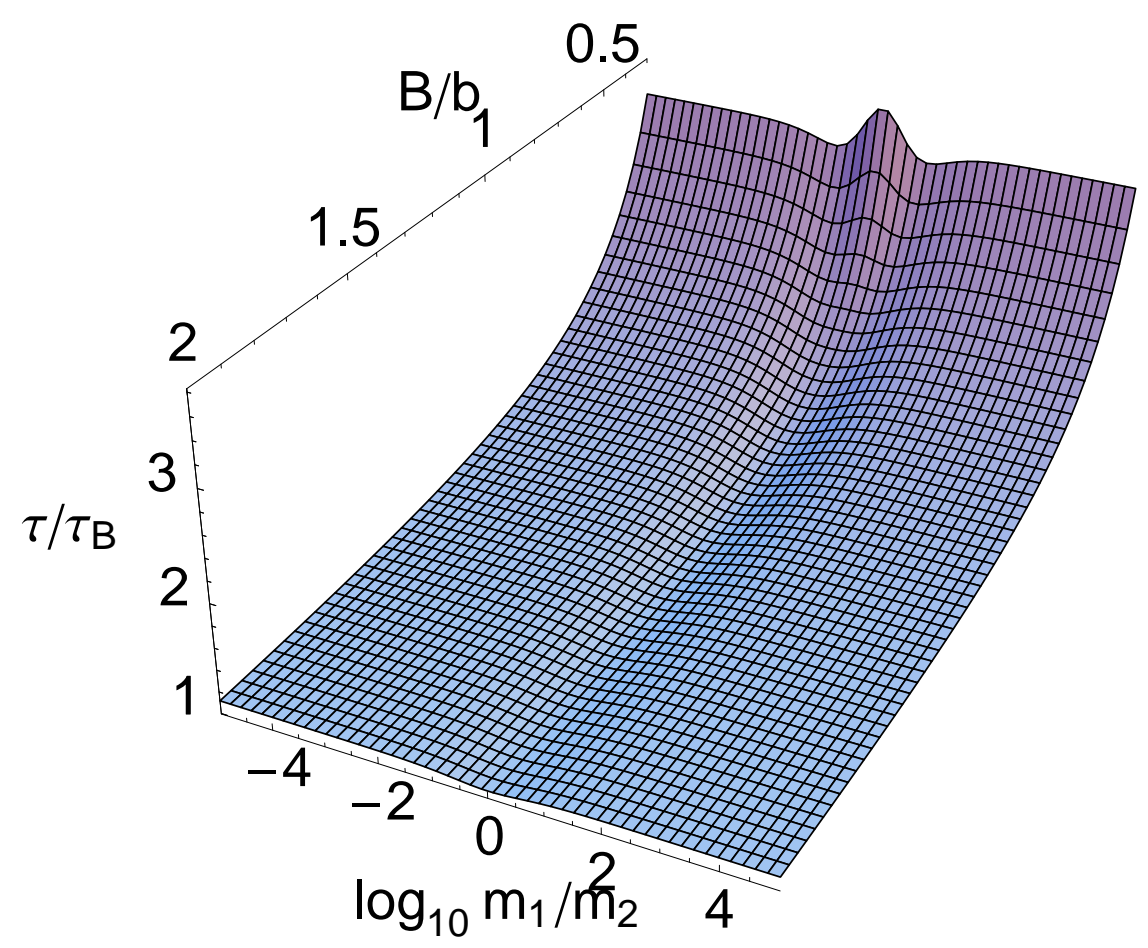

Figure 4: Overall view of the $\tau / \tau_{B}$ landscape as a function of $\log _{10} m_{1} / m_{2}$ and $B / b$. Note the relation to the features of the corresponding landscape for the initial linear entropy.

state value of the linear entropy. In particular, it is also independent of the scale setting parameter $M$, and can be written explicitly as

$$
\frac{\tau}{\tau_{B}}=\frac{b / B}{1-\Delta(0)}=\left[\left(1-\frac{b^{2}}{B^{2}} \frac{m_{1} / m_{2}}{\left(1+m_{1} / m_{2}\right)^{2}}\right)^{2}+\frac{b^{2}}{B^{2}}\right] .
$$

This implies that the main general features of the $\Delta(0)$ landscape are essentially carried over to the $\tau / \tau_{B}$ landscape, as can in fact be clearly in fig. 4. One finds here the same overall structure of valleys and ridges seen in the corresponding initial linear entropy plot, in fig. 1. A similar comparison can be made for the close-ups into the more intricate region of the $m_{1} / m_{2} \times B / b$ plane, figs. [5 and 2. As a consequence of inversion and of an additional $b / B$ factor, however, one sees that $\tau / \tau_{B}$ is bounded by unity below, while $\Delta(0)$ is similarly bounded above. For sufficiently small values of $B / b$ the characteristic time for the evolution of entanglement becomes substantially longer than $\tau_{B}$.

Note however that more tightly localized wavepackets spread faster (have smaller values of $\tau_{B}$ ), so that this does not imply very long 'absolute' times for entanglement evolution. Using the value of $M$ for scale setting, the reduced mass $\mu$ and the value of $b$ are set by the values of $m_{1} / m_{2}$ and of the oscillator frequency $\omega$, the wavepacket width $B$ being finally fixed by $B / b$. The limiting value of the 'absolute' characteristic time $\tau$ for tightly confined center of mass wavepackets fixed in this way is

$$
\lim _{B \rightarrow 0} \tau=\frac{\mu b^{2}}{\hbar},
$$

which is just the inverse of the oscillator frequency $\omega$. 


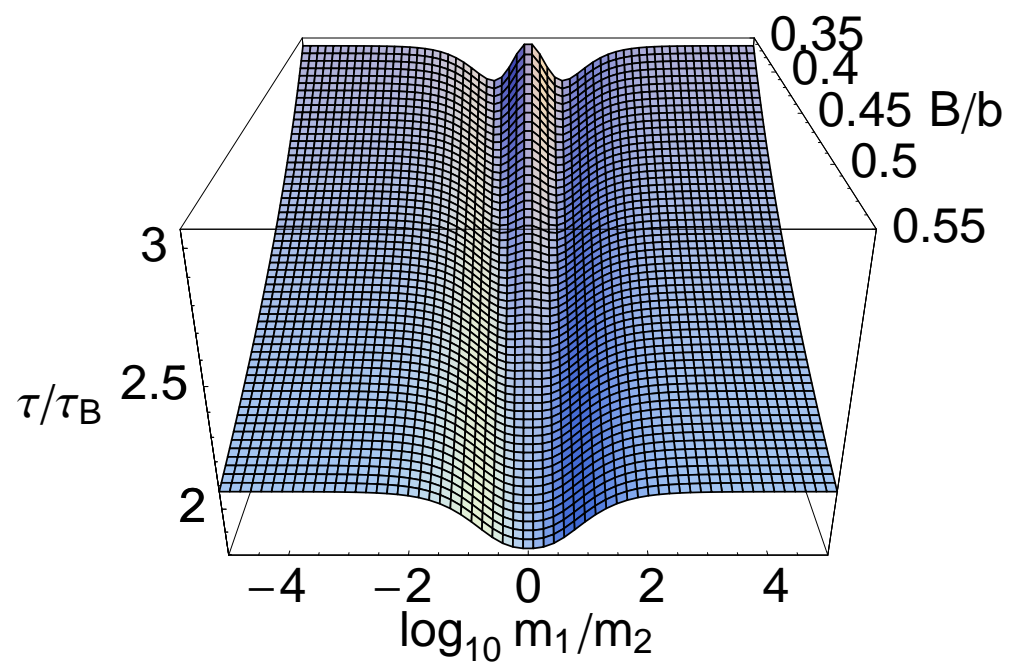

Figure 5: Closer view of the valley bifurcation at $m_{1} / m_{2}=1, B / b=0.5$ in the $\tau / \tau_{B}$ landscape. Compare with fig. 2 .

\section{Concluding remarks}

We have examined in some detail the quantum entanglement of two particles in one dimension whose center of mass evolves as a free gaussian wavepacket with mean momentum $\hbar K$, when they are bound in the ground state of a two-body harmonic interaction potential. While the fact that the occurrence of entanglement is of no surprise in view of the strong mutual correlation (e.g. in position space) of the two particles [17, one can easily see in this case that the entanglement may nevertheless be made to vanish in the initial state by an appropriate choice of the width parameter of the center of mass wavepacket. The fact that particle-particle correlations can, at least in some cases, be represented in unentagled form (which, in the present case, means being represented by factorized amplitudes) indicates that the two concepts are in fact quite distinct.

Given the stationary character of the bound state of the two particles, the dynamics of their entanglement is driven by the unitary, dispersive spreading of the center of mass wavepacket. In particular, it also comes out as being independent of the mean value of the center of mass momentum, a fact which can be understood in terms of the preservation of the coefficients in the Schmidt decomposition of the two particle wavefunction under center of mass boosts. The time dependence induced by the unitary driving dynamics on the particle-particle entanglement is algebraically akin to that governing the change in time of the width parameter of the center of mass wavepacket, but involves its own, different time scale. The two time scales become even more distinct as the initial width parameter of the center of mass wavepacket becomes appreciably smaller than the bound state size parameter. In fact, we have seen that the quadratic decrease, as $B \rightarrow 0$, of the characteristic time $\tau_{B}$ for wavepacket spreading is not followed by the characteristic time for the evolution of entanglement, which approaches a finite limit involving properties of the two particle bound state.

One must note also that the monotonic increase in time of both the spreading of the initial width $B$ of the center of mass wavepacket and of the linear entropy $\Delta$ used to measure the degree of particle-particle entanglement hinge on the particular choice made 
for the initial state. A still particular, but different choice leading to also different behavior may be devised by taking advantage of the time-reversible character of the overall unitary dynamics. In fact, one may use as an alternate initial state $\tilde{\Psi}\left(k_{1}, k_{2} ; 0\right)$, the time reversed counterpart of the state resulting from our adopted initial state (2.3) after it evolves for a time $T$, i.e.

$$
\tilde{\Psi}\left(k_{1}, k_{2} ; 0\right) \equiv \Psi^{*}\left(-k_{1},-k_{2} ; T\right) .
$$

Due to time-reversal invariance of the overall dynamics, the time evolution of this state is given as $\tilde{\Psi}\left(k_{1}, k_{2} ; t\right)=\Psi^{*}\left(-k_{1},-k_{2} ; T-t\right)$, so that the time evolution from $\Psi\left(k_{1}, k_{2} ; 0\right)$ to $\Psi\left(k_{1}, k, T\right)$ is traced backwards in time. As a result, the center of mass wavepacket will shrink and the linear entropy will decrease as $t$ increases from 0 to $T$. In particular, if $\Psi\left(k_{1}, k_{2} ; 0\right)$ is a particle-factorizable initial state, the linear entropy decreases smoothly from a non zero value to zero in the finite time $T$, as in cases of the so called entanglement sudden death [12], but immediately and also smoothly rebounds to the monotonic increase for subsequent times.

Finally, it is worth stressing that our simple model system provides for instances of quantum states consisting of parts which are strongly correlated dynamically but which are nevertheless not entangled. This is in a way complementary to the situation treated by Schrödinger [1] in the wake of the Einstein, Podolski and Rosen [2] argument, namely one in which entanglement persists after interaction between the two parts has ceased. Both cases point to a picture in which entanglement is ultimately a quantum kinematical feature, even if circumstantially affected by quantum dynamical processes.

\section{Acknowledgments}

FRP thanks Marcio Cornélio and Jonas Larson for useful discussions. This work has been supported in part by CNPq, FAPESP, and the Swedish Royal Council (Vetenskaprådet).

\section{Appendix}

We give here, for completeness, a brief, elementary calculation showing that the two reduced densities $\rho^{(i)}\left(k_{1}, k_{2}\right), i=1,2$ have the same eigenvalues and that the wavefunction $\Psi\left(k_{1}, k_{2}\right)$ can in fact be written in the form shown in eq. (3.12), cf. ref. [15], Chap. VI, section 2. We omit the time variable, which is not relevant here.

Note first that the set of eigenfunctions of $\rho^{(1)}$ can be extended to a complete orthonormal set $\left\{\chi_{n}^{(1)}\left(k_{1}\right)\right\}$ in the Hilbert space of particle 1 by including eigenfunctions with eigenvalue zero. Consider, in addition, a complete orthonormal set $\left\{u_{\nu}\left(k_{2}\right)\right\}$ in the Hilbert space of particle 2. Then, for each of the $\chi_{n}^{(1)}$ define

$$
\tilde{\chi}_{n}^{(2)}\left(k_{2}\right)=\sum_{\nu} u_{\nu}\left(k_{2}\right)\left(\chi_{n}^{(1)} u_{\nu}, \Psi\right)
$$

the bracket being the scalar product in product space. This may be checked to be an eigenfunction of $\rho^{(2)}$. In fact, write this reduced density as

$$
\rho^{(2)}\left(k_{2}, k_{2}^{\prime}\right)=\sum_{n} \int d k_{1} \int d k_{1}^{\prime} \chi_{n}^{(1) *}\left(k_{1}\right) \Psi\left(k_{1}, k_{2}\right) \Psi^{*}\left(k_{1}^{\prime}, k_{2}^{\prime}\right) \chi_{n}^{(1)}\left(k_{1}^{\prime}\right)
$$

so that 


$$
\begin{aligned}
\int d k_{2}^{\prime} \rho^{(2)}\left(k_{2}, k_{2}^{\prime}\right) \tilde{\chi}_{n}^{(2)}\left(k_{2}^{\prime}\right) & =\sum_{n^{\prime}} \int d k_{1} \chi_{n^{\prime}}^{(1) *}\left(k_{1}\right) \Psi\left(k_{1}, k_{2}\right) \sum_{\nu}\left(\Psi, \chi_{n^{\prime}}^{(1)} u_{\nu}\right)\left(\chi_{n}^{(1)} u_{\nu}, \Psi\right)= \\
& =\lambda_{n}^{(1)} \tilde{\chi}_{n}^{(2)}\left(k_{2}\right) .
\end{aligned}
$$

The sum over $\nu$ in the last expression involves the reduced density $\rho^{(1)}$ and is in fact just $\lambda_{n}^{(1)} \delta_{n n^{\prime}}$, and the integral over $k_{1}$ yields the function $\tilde{\chi}_{n}^{(2)}\left(k_{2}\right)$. This shows then that this function is an eigenfunction of the reduced density $\rho^{(2)}$ with the same eigenvalue as its parent eigenfunction $\chi_{n}^{(1)}\left(k_{1}\right)$ of $\rho^{(1)}$. From now on we may thus omit the eigenvalue superscripts.

Next check the norms of the functions $\tilde{\chi}_{n}^{(2)}$. This is easily done by evaluating

$$
\left(\tilde{\chi}_{n^{\prime}}^{(2)}, \tilde{\chi}_{n}^{(2)}\right)=\sum_{\nu}\left(\Psi, \chi_{n^{\prime}}^{(1)} u_{\nu}\right)\left(\chi_{n}^{(1)} u_{\nu}, \Psi\right)=\lambda_{n} \delta_{n n^{\prime}}
$$

showing that the functions $\chi_{n}^{(2)}\left(k_{2}\right)=\tilde{\chi}_{n}^{(2)}\left(k_{2}\right) / \sqrt{\lambda_{n}}$ constitute an orthonormal set. On the other hand, $\Psi\left(k_{1}, k_{2}\right)$ may be expanded in the product base $\left\{\chi_{n}^{(1)}\left(k_{1}\right) u_{\nu}\left(k_{2}\right)\right\}$ :

$$
\begin{aligned}
\Psi\left(k_{1}, k_{2}\right) & =\sum_{n} \sum_{\nu} \chi_{n}^{(1)}\left(k_{1}\right) u_{\nu}\left(k_{2}\right)\left(\chi_{n}^{(1)} u_{\nu}, \Psi\right)= \\
& =\sum_{n} \chi_{n}^{(1)}\left(k_{1}\right) \tilde{\chi}_{n}^{(2)}\left(k_{2}\right)=\sum_{n} \sqrt{\lambda_{n}} \chi_{n}^{(1)}\left(k_{1}\right) \chi_{n}^{(2)}\left(k_{2}\right)
\end{aligned}
$$

which establishes eq. (3.12).

\section{References}

[1] E. Schrödinger, "Discussion of Probability Relations between Separated Systems", Proceedings of the Cambridge Philosophical Society 31, 555-563 (1935).

[2] A. Einstein, B. Podolsky, and N. Rosen, "Can Quantum-Mechanical Description of Physical Reality Be Considered Complete?", Phys. Rev. 47, 777-780 (1935).

[3] J. Bell, Speakable and Unspeakable in Quantum mechanics, Cambridge University Press, 1987.

[4] A. Aspect, P. Grangier, and G. Roger, "Experimental Tests of Realistic Local Theories via Bell's Theorem", Phys. Rev. Lett. 47, 460-463 (1981).

[5] N. D. Mermin, "Bringing home the atomic world: Quantum mysteries for anybody", Am. J. Phys. 49, 940-943 (1981).

[6] R. Horodecki, P. Horodecki, M. Horodecki, and K. Horodecki, "Quantum entanglement", Rev. Mod. Phys. 81, 865-942 (2009).

[7] M. A. Nielsen and I. L. Chuang Quantum Computation and Quantum Information, Cambridge University Press 2000.

[8] John Preskill Lecture Notes for Physics 229: Quantum Information and Computation, September, 1998. 
[9] F. Mintert, A. R. R. Carvalho, M. Kus, and A. Buchleitner, "Measures and dynamics of entangled states", Phys. Reports 415, 207-259 (2005).

[10] W. H. Zurek, "Decoherence and the Transition from Quantum to Classical - Revisited", arXiv:quant-ph/0306072 (2003); Rev. Mod. Phys. 75, 715-775 (2003).

[11] R. Omns, The Interpretation of Quantum Mechanics, Princeton University Press, 1994.

[12] Ting Yu and J. H. Eberly, "Finite-Time Disentanglement via Spontaneous Emission", Phys. Rev. Lett. 93, 140404 (2004); "Sudden Death of Entanglement", Science 323, 598-601 (2009).

[13] M. P. Almeida, F. de Melo, M. Hor-Meyll, A. Salles, S. P. Walborn, P. H. Souto Ribeiro, and L. Davidovich, "Environment-Induced Sudden Death of Entanglement", Science 316, 579-582 (2007).

[14] Z.-J. Li, J.-Q. Li, Y.-H. Jin, and Y.-H. Nie, "Time evolution and transfer of entanglement between an isolated atom and a Jaynes-Cummings atom", J. Phys. B: At. Mol. Opt. Phys. 40, 3401-3411 (2007).

[15] J. von Neumann, Mathematical Foundations of Quantum Mechanics, Princeton University Press, 1955, Chap. V, section 3.

[16] H. Falk, "Inequalities of J. W. Gibbs", Am. J. Phys. 38, 858-869 (1970).

[17] P. Tommasini, E. Timmermans, and A. F. R. de Toledo Piza, "The hydrogen atom as an entangled electron-proton system", Am. J. Phys. 66, 881-886 (1998). 